\title{
Autoeficacia y estilos de afrontamiento al estrés en estudiantes universitarios
}

\author{
Self-efficacy and stress coping styles in university students \\ Lucía Florencia Piergiovanni ${ }^{1}$ \\ Pablo Domingo Depaula ${ }^{2,3}$
}

\author{
${ }^{1}$ Facultad de Psicología y Relaciones Humanas, Universidad Abierta Interamericana. Argentina \\ ${ }^{2}$ Consejo Nacional de Investigaciones Cientificas y Técnicas. Argentina \\ ${ }^{3}$ Instituto de Investigaciones en Psicología de la Universidad de Buenos Aires. Argentina
}

\begin{abstract}
Resumen: La presente investigación analizó la relación entre los niveles de Autoeficacia presentes en estudiantes universitarios y los Estilos de Afrontamiento al Estrés utilizados para hacer frente a demandas internas y externas. Se seleccionó una muestra de 126 estudiantes a quienes se les administró la Escala de Autoeficacia General, el Cuestionario de Estilos de Afrontamiento al Estrés y un cuestionario sociodemográfico. Los resultados indican que quienes presentan un mayor nivel de Autoeficacia utilizan los Estilos de Afrontamiento al Estrés Focalizado en la solución del problema y Reevaluación positiva. Por otro lado, quienes presentaron menor nivel de Autoeficacia recurren al estilo de afrontamiento Autofocalización negativa.
\end{abstract}

Palabras Clave: autoeficacia, estilos de afrontamiento al estrés, estudiantes universitarios, estrés, locus de control

\begin{abstract}
The present investigation analyzed the relationship between the levels of Self efficacy that students of the bachelor's degree on Psychology have and the Coping Styles that they use to face internal or external requests. A sample of 126 students was selected. They were given the General Self Efficacy Scale, the Stress Coping Strategies Questionnaire and a social demographic questionnaire. The results show that those students that have more Self-efficacy use the Coping Strategy Focused on solving the problem and Positive reappraisal. On the other hand, students that have lower Self-efficacy levels use the Negative self-blame way of coping with stress.
\end{abstract}

Key Words: self-efficacy, stress coping styles, university students, stress, locus control

Cómo citar este artículo:

Piergiovanni, L. F., \& Depaula, P. D. (2018). Autoeficacia y estilos de afrontamiento al estrés en estudiantes universitarios. Ciencias Psicológicas, 12(1), 17 - 23. doi: https://doi.org/10.22235/cp.v12i1.1591

Correspondencia: Lucia Florencia Piergiovanni, Anchorena 1.068, Temperley (CP. 1834), Lomas de Zamora-Buenos Aires, Argentinae-mail: luciapiergiovanni@live.com.ar; Pablo Domingo Depaula, Díaz Vélez 1.058, Depto. 1, Lomas de Zamora (CP. 1832) -Buenos Aires, Argentina-e-mail: lic_pablodepaula@yahoo.com.ar 


\section{Introducción}

A lo largo de la vida, los seres humanos atraviesan distintas etapas y con ellas se modifican los escenarios en que las mismas tienen lugar, i.e., la vivienda, el barrio, la escuela, la universidad.

Al comenzar a transitar una carrera universitaria, los estudiantes presentan diferencias respecto a sus capacidades para apropiarse de los nuevos conocimientos, al modo en el que esperan desempeñar sus habilidades y adaptarse "eficazmente" al contexto del aprendizaje (Schunk, 1989).

Bandura (1986) propone la Teoría Social Cognitiva planteando la idea de que los sujetos ejercen internamente control sobre sus pensamientos, sentimientos, motivaciones y conductas, brindándole referencias sobre las cuales sentarán las bases para percibir, regular y evaluar sus conductas.

La Autoeficacia refiere a la percepción que la persona tiene sobre su propia capacidad para lograr la actividad que se propone, en cuyo proceso los estudiantes interpretan los resultados de sus actividades y tareas académicas realizadas (Bandura, 1986).

Las creencias de Autoeficacia influyen sobre los "cursos de acción que las personas deciden seguir" (Bandura, 1997, p. 3), sobre la perseverancia, los sucesivos fracasos situacionales y la resiliencia, pudiendo limitar el afrontamiento al estrés (Bandura, 1997; Farchi, Cohen, \& Mosek, 2014). La percepción de factores estresantes a lo largo de una carrera universitaria se vincula a estados emocionales displacenteros que obligarán al sujeto a realizar una evaluación cognitiva de la situación perturbadora de su propio bienestar personal (Lazarus \& Folkman, 1986).

Según Pajares (2002), la Autoeficacia se desempeñará como una variable mediadora en el ámbito educativo a partir de los estados fisiológicos que los individuos experimenten cuando realizan cierta acción en un contexto de estrés. La tolerancia de los estímulos o demandas estresantes del ambiente académico dependerá del despliegue de una serie de esfuerzos cognitivos y conductuales llamados Estilos de Afrontamiento al Estrés (Lazarus \& Folkman, 1986; Matheny, Aycock, Pugh, Curlette, \& Silva-Canella, 1986).

Los procesos cognitivos y conductuales que implican el afrontamiento están relacionados entre sí y dependen tanto de la evaluación que realiza el sujeto de la situación como del uso de estrategias conductuales utilizadas para aliviar la carga que supone el estresor (Mok \& Tam, 2001; Richardson \& Poole, 2001). Una vez que el sujeto percibe un estímulo como amenazante aparece el afrontamiento ocupando el lugar de mediador entre aquellos eventos estresantes específicos y sus consecuencias emocionales.

Se distinguen así dos grandes categorías de modos de afrontamiento: basados en la resolución del problema objetivo, e.g., el desarrollo de estrategias cognitivas y conductuales, planear alternativas de solución, búsqueda de información, formular un plan de acción, etc.; dirigidos a la propia emoción del sujeto, relacionado a evitar o distanciarse del problema, el auto-reproche o la re-exaltación de los aspectos positivos, con el fin de modificar el modo en que el sujeto vive la situación estresante aún en aquellos casos en los que no pueda hacer nada para modificarla (Lazarus \& Folkman, 1984, 1986).

Sandín (2003) desarrolla la idea de que determinadas situaciones o condiciones vitales en una persona serían factores fundamentales al evaluar tanto el origen como las consecuencias de situaciones estresantes asociadas a la estructura social (i.e., la exclusión, la participación insatisfactoria o la no participación del sistema social), pudiendo generar malos hábitos o estilos de vida inadecuados.

Bajo este marco conceptual, Sandín y Chorot (2003) refieren al Afrontamiento como un "rasgo" o disposiciones personales desplegadas a través de Estilos de Afrontamiento al Estrés a los cuales las personas tenderán a recurrir ante la interacción entre su percepción de una situación y la situación en sí misma. Tales estilos son los siguientes: (1) Focalizado en la solución del problema, (2) Autofocalización negativa, (3) Reevaluación positiva, (4) Expresión emocional abierta, (5) Evitación, (6) Búsqueda de apoyo social, y (7) Religión (Véase Figura 1).

Estudios recientes evidencian que variables tales como e.g. la Autoeficacia real vs. la vivencia académica basada en la estrategia de estudios, varían dependiendo del tipo de carrera cursada (i.e., psicología, ingeniería y pedagogía; Borzone Valdebenito, 2017); i.e., que la Autoeficacia del estudiantado universitario dependería de factores académicos, institucionales y sociales, mayormente situacionales, de los contenidos de cada carrera (e.g., Quintero Montelongo, Pérez Córdoba, \& Correa Gutiérrez, 2009) y de las expectativas vocacionales subjetivas. 
1. Focalizado en la solución del problema: A las causas, el sujeto planea y ejecuta soluciones para afrontar la situación.

2. Autofocalización negativa: Se auto culpa, tiene sentimientos de indefensión e incapacidad, resignación, dependencia, pérdida de control y pesimismo.

3. Reevaluación positiva: Reconoce el evento estresante, pero se centra en los aspectos positivos de la situación.

4. Expresión emocional abierta: Descarga el mal humor con los demás, insulta, es hostil, irritable y se desahoga con los demás.

5. Evitación: Se concentra en otras cosas, prefiere no pensar en el problema.

6. Búsqueda de apoyo social: Identifica personas y redes de apoyo que puedan aportar al adecuado manejo de la situación estresante.

7. Religión: Acude a creencias religiosas para afrontar la situación, pues siente que ha perdido el control.

Figura 1

Estilos básicos de afrontamiento

$\mathrm{Al}$ respecto de tales observaciones, se conoce que el clima educativo (i.e., el sistema de valores institucionales y las actitudes de los docentes hacia los estudiantes, bajo la forma de demandas externas) influye sobre el sentido de aceptación optimista de la vida, el bienestar fisiológico y psicológico, y sobre los sucesos académicos de los estudiantes (Ruus, 2007), primando dichas demandas sobre las demandas internas (las últimas, más emparentadas al despliegue de los Estilos de Afrontamiento al Estrés).

Sin embargo, vale mencionar un estudio anterior realizado en el contexto argentino en una muestra de 292 sujetos adultos de población general residentes en la Ciudad Autónoma de Buenos Aires y sus alrededores (Brenlla, Aranguren, Rossaro, \& Vazquez, 2010), el que ha reportado altas correlaciones entre la Autoeficacia medida a través de la Escala de Autoeficacia General (EAG; Jerusalem \& Schwarzer, 1992; versión argentina adaptada por Brenlla et al., 2010) y algunos de los Estilos de Afrontamiento medidos a través de la Escala Breve de Afrontamiento (Brief COPE; Carver,1997; versión argentina adaptada por Brenlla \& Infante Geronimi, 2008), i.e., Planeamiento $(r=.417 ; p<.001)$, Afrontamiento activo $(r=.357 ; p<.001) \mathrm{y}$, algo inferiores, con Positivismo $(r=.215 ; p<.001)$, mostrando estos resultados una "convergencia conceptual de los constructos de autoeficacia y de los estilos de afrontamiento centrados en la tarea" (Brenlla et al., 2010; p. 84 ), por encima de la autorregulación emocional.
Brenlla et al. (2010) mostraron que a menor percepción de Autoeficacia se hallaron mayores creencias de control externo (i.e., Locus de control externo; Brenlla, Vázquez, \& Aranguren, 2008; Rotter, 1966), diferenciando Expectativas de Autoeficacia y Expectativas de Resultados acerca de una acción, siendo el primero la confianza que se tiene sobre sí mismo a la hora de realizar una acción específica, mientras que el segundo se centra en lo que se espera que una acción produzca como efecto o consecuencia una vez realizada.

Considerando los antecedentes planteados hasta aquí, el problema de investigación del presente estudio tiene como interrogante si existen relaciones entre la Autoeficacia y los Estilos de Afrontamiento al Estrés utilizados por estudiantes universitarios argentinos. Interesa conocer en qué medida el modo en que los estudiantes perciben sus propias capacidades vinculadas al logro o al fracaso académico se relaciona con alguna tipología de afrontamiento.

En suma: Los niveles de Autoeficacia de los estudiantes universitarios, ¿Se asocian con algún/ os Estilo/s de Afrontamiento en particular? A continuación, se detallará la metodología de investigación seleccionada para responder al problema de investigación planteado.

\section{Materiales y método}

\section{Diseño de investigación}

Se realizó un estudio correlacional, transversal, con la finalidad de identificar si existían relaciones entre determinadas variables en un momento dado (Hernández Sampieri, FernándezCollado, \& Baptista Lucio, 2006); i.e., se testeó la existencia de algún grado de asociación entre las siguientes variables: Autoeficacia General y Estilos de Afrontamiento al Estrés.

\section{Participantes}

Se seleccionó una muestra de tipo no probabilística e intencional integrada por 126 estudiantes de una licenciatura en Psicología dictada en una universidad privada argentina. Con respecto a la edad, la media fue de 27.91 años $(D E=8.68)$. El $19.8 \%(n=25)$ eran hombres y el $80.2(n=101)$ mujeres que se encontraban cursando alguno de los cinco años en los que se desarrolla el plan de estudios respectivo. 
Para llevar a cabo la investigación, el criterio de inclusión fue de seleccionar aquellos estudiantes de la Licenciatura en Psicología cuyas edades oscilaban entre los 18 y 60 años.

\section{Instrumentos}

A fin de evaluar las variables principales del estudio se elaboró una batería psicométrica integrada por los siguientes instrumentos:

- Cuestionario Socio-demográfico: Encuesta dirigida a indagar datos específicos de los estudiantes evaluados, i.e., su edad, sexo, estado civil, grupo conviviente, cantidad de hijos, lugar de residencia, empleo, año de cursada en el que se encontraba en el marco de la licenciatura al momento de responder a la encuesta, etc.

- Escala de Autoeficacia General (EAG) (Jerusalem \& Schwarzer, 1992; versión argentina adaptada por Brenlla, Aranguren, Rossaro, \& Vázquez, 2010): La EAG corresponde a una escala que evalúa las creencias que el examinado posee en cuanto a su propia Autoeficacia. Consta de 10 ítems de respuesta con formato de escala tipo Likert dispuesta de 4 puntos cuyos gradientes son los siguientes: Nunca, Pocas veces, Muchas veces y Siempre, a los cuales se les otorga un puntaje de 1, 2, 3 y 4 puntos respectivamente, indicando, los valores más altos, una mayor percepción de Autoeficacia. La fiabilidad de este instrumento es adecuada $(\alpha$ de Cronbach $=.76)$.

- Cuestionario de Afrontamiento al Estrés (CAE) (Sandín \& Chorot, 2003): El CAE constituye un instrumento de autoinforme que consta de 42 ítems y 7 subescalas para evaluar siete estilos básicos de afrontamiento: Por último, los coeficientes de fiabilidad para las 7 subescalas variaron entre .64 y .92 (con una media de .79).

\section{Procedimiento}

Habiendo obtenido la autorización de la institución universitaria respectiva, se administraron los cuestionarios Socio-demográfico, EAG y CAE bajo la modalidad de lápiz y papel, asegurándose a los estudiantes que los datos serían utilizados para fines exclusivamente investigativos (de modo anónimo, firmándose un consentimiento informado; en línea con los principios éticos en investigación psicológica; American Psychological Association, 1992; Richaud, 2007).

\section{Análisis de datos}

Los datos recopilados en la batería de test fueron analizados mediante el paquete estadístico SPSS (versión 22.0), utilizándose el Test de Kolmogorov-Smirnov para analizar la distribución muestral y el coeficiente estadístico Rho de Spearman para analizar correlaciones entre las variables mencionadas.

\section{Resultados}

Primeramente, a fin de verificar si las muestras respondían a una distribución normal, se llevó a cabo la prueba de normalidad a través del Test de Kolmogorov-Smirnov, evidenciando una distribución asimétrica de las variables a analizar $(p<.05)$. Con estos parámetros estadísticos, se efectuaron análisis de tipo no paramétrico.

\section{Relaciones entre los niveles de Autoeficacia y los Estilos de Afrontamiento al Estrés}

Se calculó el coeficiente Rho de Spearman hallándose una moderada asociación positiva significativa entre el nivel de Autoeficacia de los participantes y el Estilo de Afrontamiento al Estrés denominado Focalizado en la solución del proble$m a(\rho=.347 ; p=.000)$. Dicho resultado indica que cuanto mejores y más adecuados sean los juicios sobre las propias capacidades subjetivas, los estudiantes aplicarán un Estilo de Afrontamiento que implica el análisis de las causas del problema y el planeamiento y ejecución de soluciones para afrontar las situaciones estresantes.

Por otro lado, los resultados obtenidos reflejan una moderada asociación negativa significativa entre la Autoeficacia de los estudiantes y el Estilo de Afrontamiento al Estrés denominado Autofocalización negativa $(\rho=-.412 ; p=.000)$, lo que indica que ante la manifestación o elaboración de juicios precarios acerca de las propias capacidades que posee un sujeto, se aplicará un Estilo de Afrontamiento que implique ideas de autoculpabilidad, sentimientos de indefensión, dependencia y pesimismo ante situaciones estresantes. Paralelamente, se observa una escasa correlación positiva significativa entre la Autoeficacia y el Estilo de Afrontamiento al Estrés Reevaluación positiva $(\rho=.202 ; p=.023)$, lo que sugiere que el aumento del nivel de juicios positivos acerca de las propias capacidades facilitará el reconocimiento de un 
evento como estresante, centrándose el sujeto, para su resolución, en los aspectos positivos de la situación.

Tabla 1

Relaciones entre Autoeficacia y Estilos de Afrontamiento al Estrés

\begin{tabular}{|c|c|c|}
\hline \multicolumn{2}{|c|}{ Estilos de Afrontamiento al Estrés } & Autoeficacia \\
\hline \multirow{2}{*}{$\begin{array}{l}\text { Focalizado en la } \\
\text { solución del problema }\end{array}$} & $\begin{array}{l}\text { Coeficiente de } \\
\text { correlación }\end{array}$ & .347 \\
\hline & Sig. (bilateral) & .000 \\
\hline \multirow{2}{*}{$\begin{array}{l}\text { Autofocalización } \\
\text { negativa }\end{array}$} & $\begin{array}{l}\text { Coeficiente de } \\
\text { correlación }\end{array}$ & -.412 \\
\hline & Sig. (bilateral) & .000 \\
\hline \multirow{2}{*}{ Reevaluación positiva } & $\begin{array}{l}\text { Coeficiente de } \\
\text { correlación }\end{array}$ & .202 \\
\hline & Sig. (bilateral) & .023 \\
\hline \multirow{2}{*}{$\begin{array}{l}\text { Expresión emocional } \\
\text { abierta }\end{array}$} & $\begin{array}{l}\text { Coeficiente de } \\
\text { correlación }\end{array}$ & -.085 \\
\hline & Sig. (bilateral) & .346 \\
\hline \multirow{2}{*}{ Evitación } & $\begin{array}{l}\text { Coeficiente de } \\
\text { correlación }\end{array}$ & -.076 \\
\hline & Sig. (bilateral) & .400 \\
\hline \multirow{2}{*}{$\begin{array}{l}\text { Búsqueda de apoyo } \\
\text { social }\end{array}$} & $\begin{array}{l}\text { Coeficiente de } \\
\text { correlación }\end{array}$ & .052 \\
\hline & Sig. (bilateral) & .563 \\
\hline \multirow{2}{*}{ Religión } & $\begin{array}{l}\text { Coeficiente de } \\
\text { correlación }\end{array}$ & -.081 \\
\hline & Sig. (bilateral) & .365 \\
\hline
\end{tabular}

\section{Discusión y conclusiones}

De acuerdo a lo observado en la presente investigación, el aumento de la Autoeficacia en los estudiantes de una licenciatura en Psicología podría asociarse o conducir a la elección de un Estilo de Afrontamiento como es el caso del Focalizado en la solución del problema. Al recurrir a este tipo de estilo, frente a determinada situación estresante, el sujeto desarrolla estrategias cognitivas y conductuales planeando alternativas de solución para sortear adecuadamente la fuente de estrés; en este caso, las valoraciones positivas que haga la persona acerca de sus propias capacidades serán el principal procesamiento cognitivo que los hará optar por afrontar situaciones orientando sus actos a solucionar los problemas que le originen estrés. Así, sujetos cuyas creencias de Autoeficacia sean elevadas, serán capaces de sostener y aplicar oportuna y eficazmente el Estilo de Afrontamiento empleado para alcanzar la meta propuesta; el énfasis será puesto en la resolución del problema y debido a su alto nivel de Autoeficacia, posiblemente la persona emplee el tiempo que sea necesario y utilice sus energías a fin de encontrar la solución más efectiva.

Por otro lado, considerando la asociación negativa significativa hallada entre la Autoeficacia y el Estilo de Afrontamiento Autofocalización negativa, se estima que aquellos estudiantes que tienden a valorar negativamente sus propias capacidades para llevar a cabo determinada acción, al momento de decidir de qué modo hacer frente a una situación que les genere estrés, optarán por un recurso que los llevará a auto-culparse por la situación, generándose un sentimiento de indefensión o incapacidad, o bien la persona podrá resignarse ante el problema. En este caso, la función de mediador cognitivo que presenta la Autoeficacia será insuficiente para que el sujeto logre alcanzar una meta propuesta, ya que debido a la valoración negativa que hace de sus propias capacidades, no podrá desplegar otras estrategias para salir lo menos perjudicado posible de tal situación estresante.

Otro de los resultados obtenidos se vincula al hecho de que los sujetos que se perciben con mayor capacidad para llevar a cabo una acción al momento de elegir un recurso para afrontar situaciones estresantes, optan por reconocer el evento que les genera estrés pero centrándose en los aspectos positivos de la situación, i.e., mediante el Estilo de Afrontamiento Reevaluación positiva. Esto último resulta esperable por considerar a la Autoeficacia como mediadora cognitiva entre el sujeto y las tareas que se propone; en casos como este, el sujeto logra reconocer una parte positiva dentro de una situación que él mismo considera sobrepasa sus exigencias o recursos, principalmente gracias a esa creencia que posee acerca de sus capacidades, i.e., su Autoeficacia. A diferencia de lo apuntado en el párrafo anterior, altos niveles de Autoeficacia producto de logros académicos reiterados, permiten revalorizar las situaciones de estrés como un desafío para incrementar la eficacia personal (Bandura, 1999, 2002) revalorizando óptimamente las situaciones de estrés. Vale aclarar que si bien la correlación hallada entre la Autoeficacia y el Estilo de Afrontamiento Reevaluación positiva fue moderada tendiendo a ser baja, i.e., alcanzando un valor de coeficiente $\rho=.202$ 
$(p=.023)$, resulta aceptable, en tanto, es cercana a la "mediana" según la clasificación de tamaños del efecto de Cohen (1992; Meyer et al., 2001).

La variable Autoeficacia ha sido analizada en estudios anteriores conjuntamente con el constructo denominado Locus de control, este último definido como una variable cognitiva que refiere a la creencia que una persona tiene respecto a la relación entre su conducta y las consecuencias de la misma (Visdómine-Lozano \& Luciano, 2006). Este concepto cuenta con dos aspectos, por un lado el Locus de control interno, que implica la creencia de que el resultado de la conducta de la persona está directamente relacionado con sus comportamientos individuales; y el Locus de control externo, que involucra la creencia de que factores ajenos y externos a la persona explicarían o controlarían las acciones. La relación entre la variable mencionada y la Autoeficacia refiere a la influencia de ambas respecto a la experiencia subjetiva de las personas, aludiendo que tanto una como la otra en interacción con el medio determinarían la iniciación y la persistencia de algunas de las conductas individuales, motivos por los cuales se les atribuye a ambos factores cognitivos la capacidad de regular el funcionamiento individual (Moretti, Medrano, \& Basler, 2015).

En relación a los resultados obtenidos en el presente estudio, resulta oportuno y coherente establecer una relación hipotética entre aquellos y el constructo Locus de control interno recientemente definido. Los estudiantes de la muestra analizada presentaron relaciones significativas en cuanto a sus niveles de Autoeficacia y los Estilos de Afrontamiento al Estrés que utilizan ante situaciones que consideran extremas o que sobrepasan sus capacidades. Si se considera el nivel de Autoeficacia presente en un sujeto como un potencial mediador cognitivo interviniente en la planificación de respuestas ante las demandas internas o propias del medio ambiente, es lícito concluir que en los casos en los que los sujetos utilizan los Estilos de Afrontamiento al Estrés Focalizado en la solución del problema, Autofocalización negativa y Reevaluación positiva estarían eventualmente llevando a cabo un procesamiento cognitivo en el cual se reconoce el propio sujeto como responsable de sus propios actos o de la "suerte" y las consecuencias de los mismos, esforzándose por solucionar la situación conflictiva, auto-culpándose por lo sucedido, o bien, extrayendo una parte positiva aún de las experiencias displacenteras.
En línea con lo inferido en el párrafo anterior, el presente estudio resulta coincidente con los hallazgos de Brenlla et al. (2010), en tanto los sujetos responden al estrés basándose en alguno de sus estilos individuales de afrontamiento, modificando subjetivamente sus creencias internas, superando tales procesos cognitivos a la focalización dirigida hacia los factores situacionales objetivos (i.e., demandas externas), en los que hacían énfasis otros estudios ya comentados (e.g., Borzone Valdebenito, 2017; Quintero Montelongo et al., 2009; Ruus, 2007). Esta línea argumental basada en los resultados de la presente investigación resultaría coincidente con los hallazgos de Brenlla et al. (2010), en tanto, las autoras concluyeron que conforme se incrementa el nivel de Autoeficacia disminuyen las creencias relacionadas con el Locus de control externo, y las conductas centradas en la emoción (i.e., la aplicación de Estilos de Afrontamiento al Estrés de prevalencia afectiva, e.g. Expresión emocional abierta, Evitación, Búsqueda de apoyo social, y que por ende difieren a los estilos Focalizado en la solución del problema, Autofocalización negativa y Reevaluación positiva, netamente cognitivos). En lo que respecta a las limitaciones del estudio, se reconoce que los coeficientes de correlación obtenidos fueron medio-bajos o moderados, más allá de los altos niveles de significación alcanzados $(p=.000)$; especialmente, el coeficiente de correlación más bajo lo representó la asociación hallada entre la Autoeficacia y el Estilo de Afrontamiento al Estrés Reevaluación positiva ( $\rho=.202$; $p=.023$ ), pero se considera aceptable dicho resultado por cuestiones clasificatorias del tamaño del efecto (Cohen, 1992; Meyer et al., 2001) ya discutidas arriba. Asimismo, existieron ciertas limitaciones de los instrumentos psicométricos utilizados, reconociendo valores bajos de fiabilidad en algunos subíndices del CAE, aunque globalmente, este instrumento y la EAG arrojaron valores de $\alpha$ de Cronbach cercanos a .80. Para futuros estudios, podría considerarse la realización de nuevos análisis factoriales (i.e., exploratorios y confirmatorios) procurando re-testear en muestras diversas la validez y confiabilidad de ambas pruebas.

Para finalizar, el ámbito universitario puede considerarse un ambiente hostil y amenazante para algunos sujetos que en él ingresan o que lo transcurren. Por tanto, conocer los niveles de Autoeficacia presentes en los estudiantes y establecer relaciones entre dicho aspecto cognitivo y los Estilos de Afrontamiento al Estrés, resulta de suma im- 
portancia en la vida académica institucional, dado que habitualmente situaciones nuevas o extremadamente demandantes serán fuentes generadoras de estrés para el estudiantado, y de cuyas estrategias de afrontamiento instrumentadas dependerá el éxito académico posterior. Con todo, futuras investigaciones deberán considerar recursos de promoción o mejora de las estrategias académicas destinadas a aumentar los niveles de Autoeficacia y así disminuir el nivel de deserción a lo largo de la carrera. Desde otra mirada, el conocimiento acerca de los Estilos de Afrontamiento al Estrés que en el ámbito universitario se utilizan puede considerarse predictor de futuras conductas; los alumnos deben ser reforzados e incentivados a utilizar los recursos que tengan disponibles para hacer del trayecto universitario una experiencia positiva y lograr la meta propuesta.

\section{Referencias}

American Psychological Association (1992). Ethical principles of psychologist and code of conduct. American Psychologist, 47, 1597-1611.

Bandura, A. (1997). Self-efficacy: The exercise of control. New York, NY: Freeman.

Bandura, A. (1999). (Ed.), Auto-Eficacia: Cómo afrontamos los cambios dela Sociedad actual. Bilbao: Desclée de Brouwer.

Bandura, A. (2002). Social cognitive theory in cultural context. Applied Psychology: An International Review, 51, 269290. doi: 10.1111/1464-0597.00092

Bandura,A.(1986). Socialfoundations of thought andaction: Asocial cognitive theory. Englewood Cliffs, NJ: Prentice Hall.

Borzone Valdebenito, M. A. (2017). Autoeficacia y vivencias académicas en estudiantes universitarios. Acta Colombiana de Psicología, 20(1), 266-274. doi: 10.14718/ACP.2017.20.1.13

Brenlla, M. E., \& Infante Geronimi, C. (2008). Adaptación Argentina de la Escala Breve de Afrontamiento al Estrés de Carver. Manuscrito no publicado.

Brenlla, M. E., Aranguren, M., Rossaro, M. F., \& Vázquez, N. (2010). Adaptación para Buenos Aires de la Escala de Autoeficacia General. Interdisciplinaria, 27(1), 77-94.

Brenlla, M. E., Vázquez, N., \& Aranguren, M. (2008). Adaptación Argentina de la Escala de Locus de Control de Rotter [Argentinianadaptation of Rotter's Locus of ControlScale]. Manuscrito no publicado.

Carver, C. S. (1997). You want to measure coping but your protocol's too long: Consider the BRIEF-COPE. International Journal of Behavioral Medicine, 4(1), 92-100.

Cohen, J. (1992). A power primer. Psychological Bulletin, 112(1), 155-159. doi: 10.1037/0033-2909.112.1.155

Farchi, M., Cohen, A., \& Mosek, A. (2014). Developing Specific Self-Efficacy and Resilience as First Responders among Students of Social Work and Stress and Trauma Studies. Journal of Teaching in Social Work, 34, 129146. doi: $10.1080 / 08841233.2014 .894602$

Hernández Sampieri, R., Fernández-Collado, C., \& Baptista Lucio, P. (2006). Metodología de la investigación. México: McGraw-Hill.
Jerusalem, M., \& Schwarzer, R. (1992). Self-efficacy as a resource factor in stress appraisal processes. In R. Schwarzer (Ed.), Self-efficacy: Thought control of action (pp. 195-213). Washington, DC: Hemisphere.

Lazarus, R. S., \& Folkman, S. (1987). Transactional theory and research on emotions and coping. European Journal of Personality, 54, 385-405.

Lazarus, R.S., \& Folkman, S. (1986). Estrés y procesos cognitivos. Barcelona: Editorial Martínez Roca.

Lazarus, R.S., \& Folkman, S. (1984). Stress, Appraisal and Coping. New York: Springer Publishing Company.

Matheny, K.B., Aycock, D.W., Pugh, J.L., Curlette, W.L., \& Silva-Cannella, K.A. (1986). Stress Coping: A Qualitive and Quantitive Synthesis with Implications for Treatment. Counselling Psychologist, 14, 499-549.

Meyer, G. J., Finn, S. E., Eyde, L. D., Kay, G. G., Moreland, K. L., Dies, R. R., . . . Reed, G. M. (2001). Psychological testing and psychological assessment: A review of evidence and issues. American Psychologist, 56(2), 128-165. doi: 10.1037/0003-066X.56.2.128

Mok, E., \& Tam, B. (2001). Stressors and Coping Methods among Chronic Haemodialysis Patients in Hong Kong. Journal of Clinical Nursing, 10, 503-511.

Moretti, L.S., Medrano, L.A., \& Basler, H. (2015). Validación del Cuestionario de Lugar de Control de Dolor en estudiantes argentinos con cefaleas recurrentes. Pensamiento Psicológico, 13(1), 27-38. doi: 10.11144/ Javerianacali.PPSI13-1.vclc

Pajares, F. (2002). Overview of Social Cognitive Theory and Self-Efficacy. EE.UU: Emory University.

Quintero Montelongo, M. A., Pérez Córdoba, E., \& Correa Gutiérrez, S. (2009). La relación entre la autoeficacia y la ansiedad ante las ciencias en estudiantes del nivel medio superior. Revista Internacional de Ciencias Sociales y Humanidades, 19(2), 69-91.

Richardson, C., \& Poole, H. (2001). Chronic pain and coping: a proposed role for nurses and nursing models. Journal of Advanced Nursing, 34, 659-667. doi: 10.1046/j.13652648.2001.01795.x

Richaud, M. C. (2007). La ética en la investigación psicológica. Enfoques, 19, (1-2), 5-18.

Rotter, J. B. (1966). Generalized expectancies for internal versus external control of reinforcement. Psychological Monographs, 80, 1-28.

Ruus, V., Veisson, M., Leino, M., Ots, L., Pallas, L., Sarv, E., \& Veisson, A. (2007). Students' Well-Being, Coping, Academic Success, and School Climate. Social Behavior and Personality, 35(7), 919-936. doi: https:// doi.org/10.2224/sbp.2007.35.7.919

Sandín, B. (2003). El estrés: un análisis basado en el papel de los factores sociales. Revista Internacional de Psicología clínica y de la salud, 3(1), 141-157.

Sandín, B., \& Chorot, P. (2003) Cuestionario de afrontamiento del estrés (CAE): desarrollo y validación preliminar. Revista de Psicopatología y Psicología Clínica, 8(1), 39-54.

Schunk, D.H. (1989). Self-efficacy and Achievement Behaviors. Educational Psychology Review, 1(3), 173207. doi: 10.1007/BF01320134

Visdómine-Lozano, J.C., \& Luciano, C. (2006). Locus de control y autorregulación conductual: revisiones conceptual y experimental. International Journal of Clinical and Health Psychology, 6, 729-751. 